https://helda.helsinki.fi

\title{
Akkermansia muciniphila induces gut microbiota remodelling and controls islet autoimmunity in NOD mice
}

\section{Hänninen, Arno}

2018-08

Hänninen , A , Toivonen , R , Pöysti , S , Belzer , C , Plovier , H , Ouwerkerk , J P , Emani , R , Cani , P D \& De Vos , W M 2018 , ' Akkermansia muciniphila induces gut microbiota remodelling and controls islet autoimmunity in NOD mice ' , Gut, vol. 67 , no. 8 , pp. 1445-1453 . https://doi.org/10.1136/gutjnl-2017-314508

http://hdl.handle.net/10138/243585

https://doi.org/10.1136/gutjnl-2017-314508

publishedVersion

Downloaded from Helda, University of Helsinki institutional repository.

This is an electronic reprint of the original article.

This reprint may differ from the original in pagination and typographic detail.

Please cite the original version. 


\title{
Akkermansia muciniphila induces gut microbiota remodelling and controls islet autoimmunity in NOD mice
}

\author{
Arno Hänninen, ${ }^{1,2}$ Raine Toivonen, ${ }^{1}$ Sakari Pöysti, ${ }^{1}$ Clara Belzer, ${ }^{3}$ Hubert Plovier, ${ }^{4}$ \\ Janneke P Ouwerkerk, ${ }^{3}$ Rohini Emani, ${ }^{1}$ Patrice D Cani, ${ }^{4}$ Willem M De Vos ${ }^{3,5}$
}

\begin{abstract}
Additional material is published online only. To view please visit the journal online (http://dx.doi.org/10.1136 gutjnl-2017-314508).
\end{abstract}

\section{Department of Medical} Microbiology and Immunology, University of Turku, Turku, Finland

${ }^{2}$ Turku University Hospital, Hospital District of Southwest Finland, Turku, Finland ${ }^{3}$ Laboratory of Microbiology, Wagenigen University

Wageningen, The Netherlands ${ }^{4}$ Metabolism and Nutrition Research Group, Louvain Drug Research Institute, WELBIO (Walloon Excellence in Life sciences and BIOtechnology), Universite Catholique de Louvain, Brussels, Belgium ${ }^{5}$ RPU Immunobiology, Department of Bacteriology and Immunology, University of Helsinki, Helsinki, Finland

\section{Correspondence to}

Adj. professor Arno Hänninen, Department of Medical Microbiology and Immunology, University of Turku, Turku 20520, Finland; arno. hanninen@utu.fi

Received 16 May 2017 Revised 17 November 2017 Accepted 21 November 2017 Published Online First 21 December 2017

\section{Linked}

- http://dx.doi.org/10.1136/ gutjnl-2017-315732

Check for updates

To cite: Hänninen A Toivonen R, Pöysti S, et al. Gut 2018:67:1445-1453.

\section{ABSTRACT \\ Objective Intestinal microbiota is implicated in the} pathogenesis of autoimmune type 1 diabetes in humans and in non-obese diabetic (NOD) mice, but evidence on its causality and on the role of individual microbiota members is limited. We investigated if different diabetes incidence in two NOD colonies was due to microbiota differences and aimed to identify individual microbiota members with potential significance.

Design We profiled intestinal microbiota between two NOD mouse colonies showing high or low diabetes incidence by $16 \mathrm{~S}$ ribosomal RNA gene sequencing and colonised the high-incidence colony with the microbiota of the low-incidence colony. Based on unaltered incidence, we identified a few taxa which were not effectively transferred and thereafter, transferred experimentally one of these to test its potential significance.

Results Although the high-incidence colony adopted most microbial taxa present in the low-incidence colony, diabetes incidence remained unaltered. Among the few taxa which were not transferred, Akkermansia muciniphila was identified. As A. muciniphila abundancy is inversely correlated to the risk of developing type 1 diabetes-related autoantibodies, we transferred $A$. muciniphila experimentally to the high-incidence colony. A. muciniphila transfer promoted mucus production and increased expression of antimicrobial peptide $\operatorname{Reg} 3 \gamma$, outcompeted Ruminococcus torques from the microbiota, lowered serum endotoxin levels and islet toll-like receptor expression, promoted regulatory immunity and delayed diabetes development.

Conclusion Transfer of the whole microbiota may not reduce diabetes incidence despite a major change in gut microbiota, but single symbionts such as $A$. muciniphila with beneficial metabolic and immune signalling effects may reduce diabetes incidence when administered as a probiotic.

\section{INTRODUCTION}

The composition of the intestinal microbiota has been found to differ between healthy subjects and those who develop autoimmune diseases, including type 1 diabetes (T1D). ${ }^{1}$ The abundance of certain intestinal Bacteroides species has been associated with increased risk to develop diabetes-related autoantibodies in young children genetically at risk of T1D. ${ }^{2}$ In addition, a decline in the overall

\section{Significance of this study}

What is already known on this subject?

- Gut microbiota in individuals at risk to develop type 1 diabetes (T1D) differs from that of healthy individuals.

- Initial findings in humans indicate reduced bacterial diversity and dominance of certain phyla.

- In non-obese diabetic (NOD) mice, differences in diabetes incidence related to, for example, gender or defects of innate immune signalling $\left(M y D 88^{-/-}\right)$appear to be mediated by differences in gut microbiota.

- Akkermansia muciniphila is a symbiont which can induce innate immune signalling (via TLR2), supports mucus production and is an abundant constituent of microbiota in health but not in T1D or IBD.

What are the new findings?

- Although high diabetes incidence in NOD/ Jax mice was found to associate with reduced bacterial diversity, it remained high also after diversity was restored via cohousing or oral microbiota transfer.

- A. muciniphila was detected among the few taxa which were absent from NOD/Jax microbiota even after reconstitution, and when cultures of this symbiont were transferred to NOD/Jax mice via oral route, diabetes incidence was delayed significantly.

- A. muciniphila induced mucus production and expression of Reg3 $\gamma$ in colon, caused microbiota remodelling, lowered serum endotoxin levels and islet toll-like receptor expression, and promoted Foxp ${ }^{+}$regulatory T cells in islets and interleukin 10 and transforming growth factor beta in pancreatic lymph nodes.

How might it impact on clinical practice in the foreseeable future?

- Our findings suggest that $A$. muciniphila, whose administration to humans appears to be safe, is a potential candidate for clinical trials aimed at preventing T1D. 

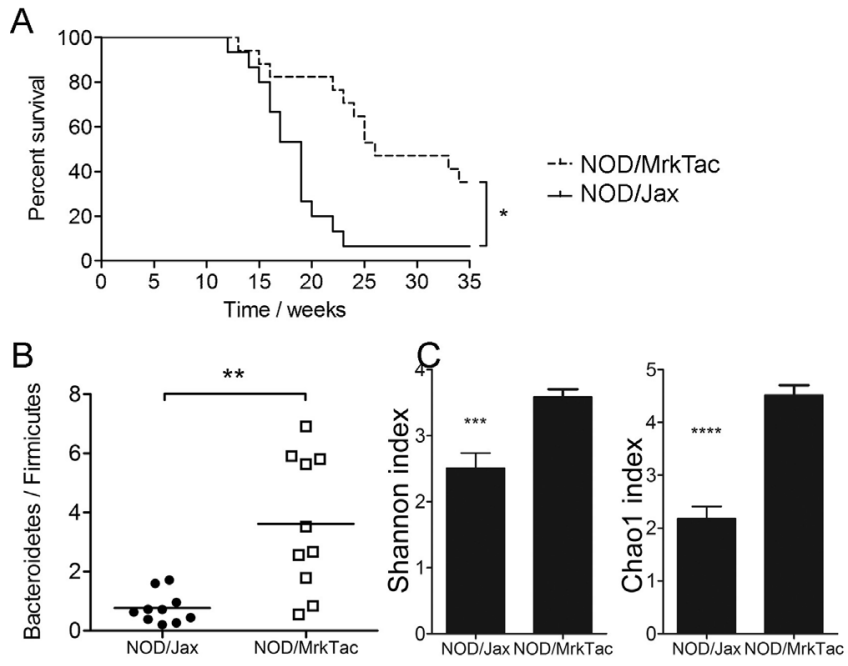

D

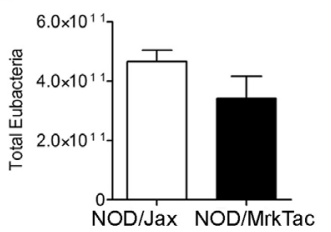

E

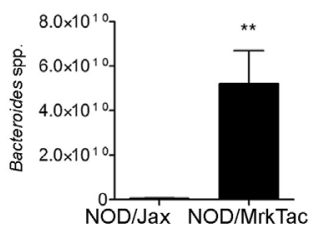

$\mathrm{F}$

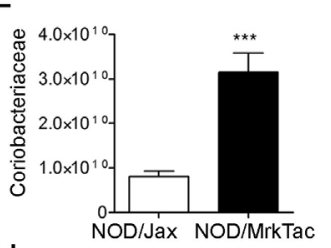

$\mathrm{H}$

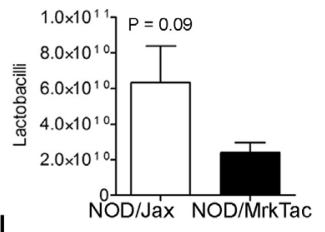

G

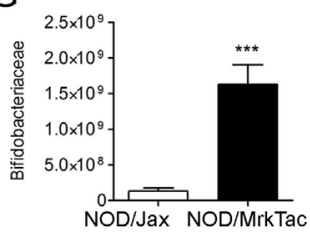

|
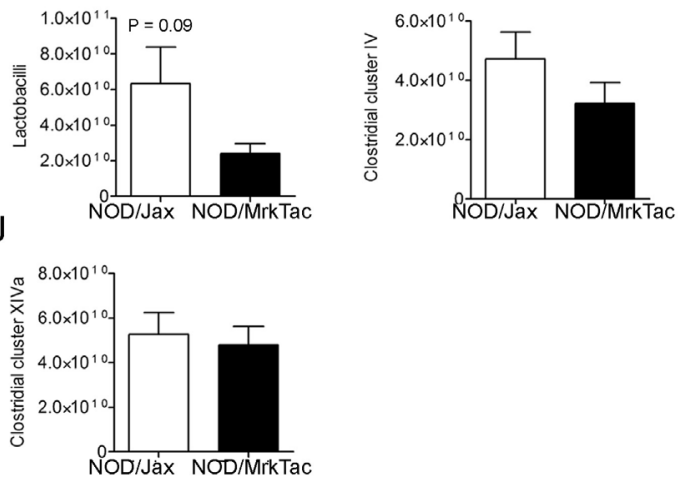

Figure 1 Two NOD colonies with different diabetes incidence differ in their microbiota composition. (A) Diabetes-free survival of female mice in the NOD/MrkTac and NOD/Jax mouse colonies ( $n=17$ mice/ group). (B) Bacteroidetes-to-Firmicutes ratio and (C) alpha diversity and Chao1 indices in NOD/MrkTac and NOD/Jax microbiota. (D-J) Initial characterisation of microbiota in NOD/Jax and NOD/MrkTac mice using quantitative PCR. Regions of 165 ribosomal RNA genes were amplified using primers specific for indicated groups. Units represent copies/mg colon and caecum content in 7-week-old donors. Data are as mean \pm SEM and consist of 10 mice/group $(B, C)$ or six mice/group (D-J). ${ }^{*} \mathrm{P}<0.05,{ }^{*} \mathrm{P}<0.01$. NOD, non-obese diabetic mice.

diversity of microbiota was found to associate with an increased risk to develop clinical disease. ${ }^{3}$ Some of the proposed mechanisms relate to local immune homeostasis and gut barrier integrity, ${ }^{45}$ activation of the adaptive immune responses interfering with peripheral tolerance to tissue antigens such as beta-cell antigens $^{6}$ or immune suppression driven by lipopolysaccharide (LPS) produced by specific gut bacteria. ${ }^{7}$ However, a causal relation between intestinal microbes and T1D has not been established, and hence, the ways in which the intestinal microbiota may affect T1D development are mostly speculative.

Preclinical studies have focused on non-obese diabetic (NOD) mice, which have developed into the archetype model for T1D. Diabetes development in NOD mice depends on gut microbiota composition and on the intestinal signalling via LPS involving TLR4 and MyD88. ${ }^{8}$ Recently, a comparison of microbiota between a NOD colony and a colony of the diabetes-resistant sister strain non-obese diabetes-resistant (NOR) mice showed differences in their species composition, and faecal transplantation of microbiota from the NOD colony induced insulitis in non-obese diabetes-resistant (NOR) hosts suggesting that NOD microbiota may carry diabetogenic effects in itself. ${ }^{9}$ However, diabetes incidence varies considerably between NOD mouse colonies around the world, suggesting that colonyto-colony variation in diabetes incidence may be due to immune modulating effects of microbiota colonisation of the gut. ${ }^{1011} \mathrm{In}$ addition to the role of microbiota in local immune responses in the intestine, individual members of gut microbiota have been found to promote T-cell responses, which may have a bearing on immune responses even beyond the intestine. ${ }^{12}$ This notion has gained lots of credence after a number of studies in which various bacteria have been identified to be able to promote regulatory $\mathrm{T}$ cells (Treg), antimicrobial or inflammatory Th17, and regulatory $\operatorname{Tr} 1$ immunity, including members of the clostridial clusters IV and XIVa, ${ }^{1314}$ segmented filamentous bacteria ((SFB), also known as Candidatus arthromitus) and mucus-adhering commensals, such as a series of Firmicutes, ${ }^{15} 16$ some species of the genus Bacteroides ${ }^{17} 18$ and the mucus-degrader Akkermansia muciniphila. ${ }^{1920}$ Differences in abundances of some of these and other potentially important bacterial taxa have been associated with the risk of developing T1D-related autoantibodies also in humans. $^{21-23}$

Here, we compared two NOD colonies with high and low diabetes incidence that differed in their intestinal microbiota. Unexpectedly, diabetes incidence was found to be resistant to microbial transfer, and hence, we focused on the taxa that were not effectively transferred. One such taxon was A. muciniphila, a mucus-using symbiont with beneficial metabolic and immune-signalling properties, which was recently found to express an outer membrane protein that is capable of signalling via the TLR2 receptor. ${ }^{24-26}$ Experimental transfer of A. muciniphila significantly delayed diabetes incidence in treated mice and showed local and systemic beneficial effects, including lowered serum endotoxin levels, reduced TLR expression and increased Foxp $3^{+}$ regulatory $\mathrm{T}$ cells in islets, and increased interleukin (IL)-10 and transforming growth factor beta (TGF- $\beta$ ) in pancreatic lymph nodes.

\section{RESULTS}

\section{Microbiota of reduced diversity associates with high incidence of diabetes}

One characteristic of a healthy microbiota able to tolerate environmental perturbations is microbial diversity, contributed by a high richness of different taxa. To compare the intestinal bacterial diversity of taxa between NOD/Jax mice and NOD/MrkTac mice, which differ considerably in their diabetes incidence (figure 1A), we used sequencing of $16 \mathrm{~S}$ ribosomal (r)RNA amplicons (online supplementary figure S1) and quantitative PCR. $\mathrm{NOD} / \mathrm{Jax}$ mice with a diabetes incidence of over $90 \%$ by 25 
A

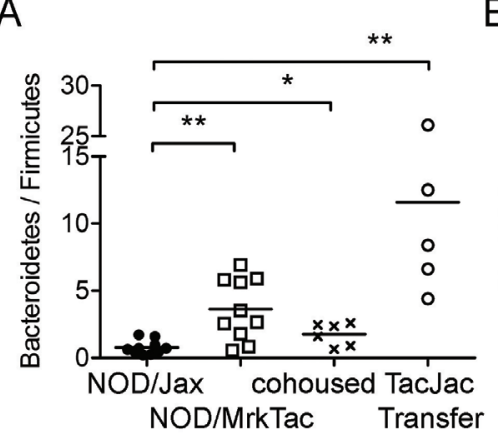

D
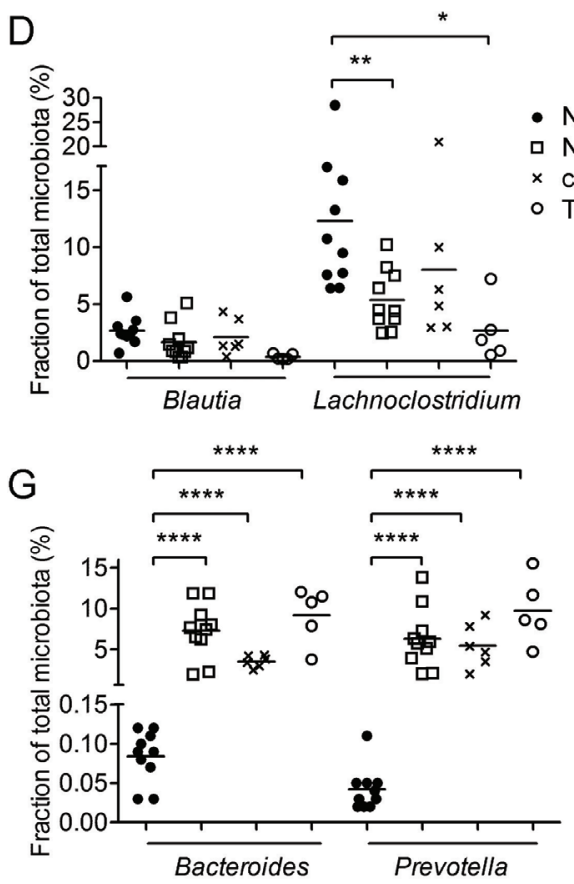

I

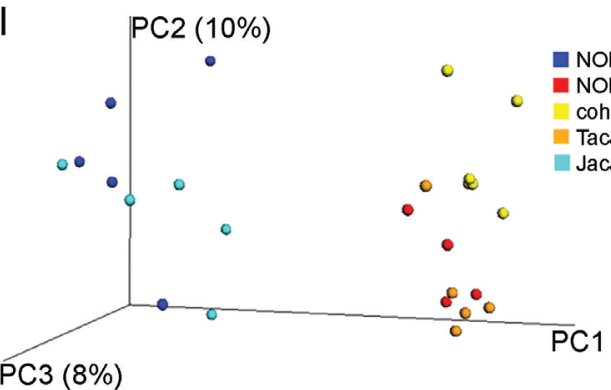

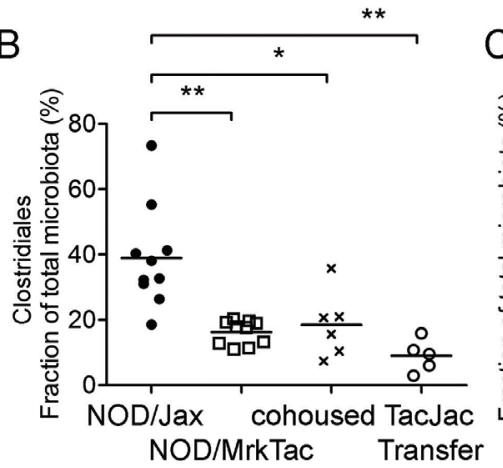

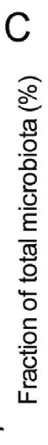

- NOD/Jax

- NOD/MrkTac

$x$ cohoused

- TacJac transfer
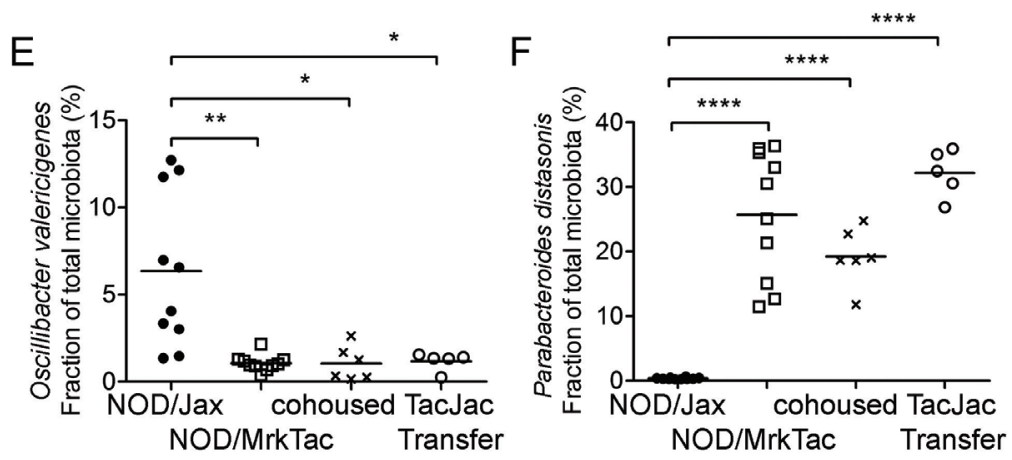

$\mathrm{H}$

- NOD/Jax

- NOD/MrkTac

$x$ cohoused

- TacJac transfer

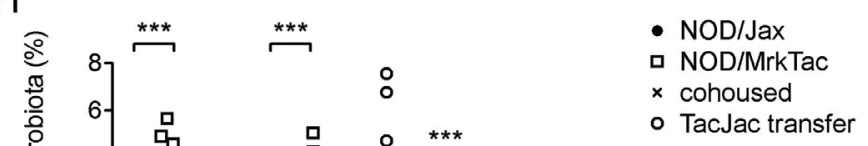

Figure 2 Colonisation of NOD/Jax mice with NOD/MrkTac microbiota does not rescue NOD/Jax mice from early diabetes development. (A) Bacteroidetes-to-Firmicutes ratio, (B) relative abundancy of Clostridiales and (C) most prominent genera within Clostridiales show microbiota adaptation, including Ruminococcus, Ethanoligenens, Faecalibacterium and Ruminoclostridium spp. (D) Relative abundancy of Blautia and Lachnoclostridium and (E) Oscillibacter valericigenes in NOD/Jax mice following cohousing and oral microbiota transfer. (F) Relative abundancy of Parabacteroides distasonis, (G) Bacteroidetes and Prevotellae and (H) indicated species within genus Bacteroidetes, including Bacteroides thetaiotaomicron and Bacteroides fragilis. (I) Principal component (PC) analysis of NOD/MrkTac, NOD/Jax and NOD/Jax mice following cohousing and oral microbiota transfer (mean \pm SD). Neither cohousing $(J)$ nor oral microbiota transfer (K) delay diabetes development in NOD/Jax mice $(n=15$ mice/ group). In (A)-(I), each symbol represents an individual mouse tested. Mice were taken from the same experiments in which mice were also followed for diabetes development at 7 weeks of age. ${ }^{*} P<0.05 ;{ }^{* *} P<0.01{ }^{* * *} P<0.001 ;{ }^{* * *} P<0.0001$. NOD, non-obese diabetic mice.

weeks of age (approximately 50\% at 18 weeks) showed significantly lower Bacteroidetes-to-Firmicutes ratio and alpha diversity (figure $1 \mathrm{~B}, \mathrm{C}$ ) compared with $\mathrm{NOD} / \mathrm{MrkTac}$ mice with a diabetes incidence that was significantly lower and reflected later diabetes onset, with only $50 \%$ of mice having diabetes at 25 weeks age.

We validated the $16 \mathrm{~S}$ rRNA results by quantitation of six genera based on amplification of bacterial DNA using 


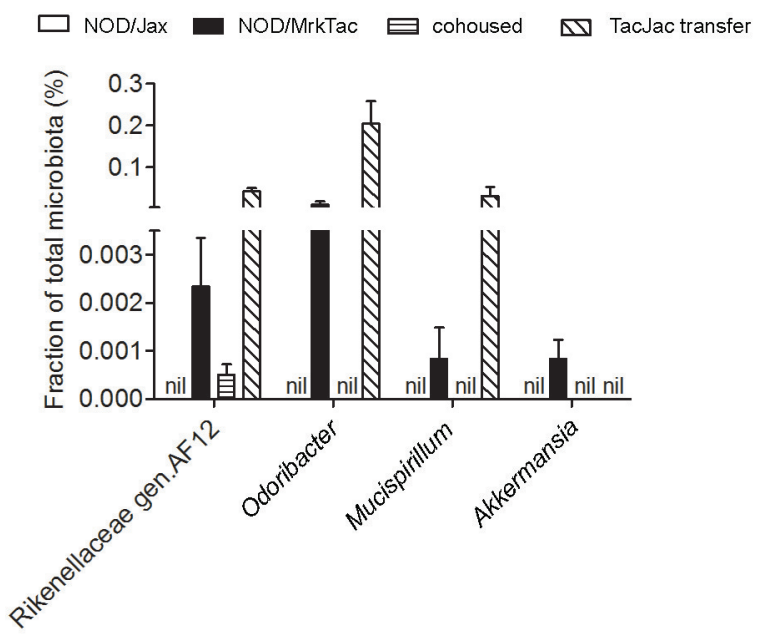

Figure 3 Identification of a few microbial taxa not efficiently transferred to NOD/Jax mice. Note that Akkermansia is not transferred following either cohousing or oral transfer. Results are representative of $16 \mathrm{~S}$ ribosomal RNA sequencing data ( $\mathrm{n}=6$ mice per group). Mice were taken from the same experiments in which mice were also followed for diabetes development at 7 weeks of age. Data are as mean \pm SEM. NOD, non-obese diabetic mice.

genus or group-specific primers. According to these data, the low abundance of Bacteroidetes in NOD/Jax mice in $16 \mathrm{~S}$ rRNA data is partly explained by a very low abundance of Bacteroides spp, as primers specific for Bacteroides spp amplified DNA almost exclusively from NOD/MrkTac mice (figure $1 \mathrm{D}, \mathrm{E}$ ). In addition, NOD/Jax mice showed significantly lower numbers of Coriobacteriaceae and Bifidobacteriaceae, the latter of which contain many species likely to be beneficial and are part of healthy microbiota. Interestingly, amplification of DNA using group-specific primers suggested that numbers of Clostridia cluster IV and cluster XIVa, which contain bacteria important for regulatory $\mathrm{T}$ cell generation in the colon, were equally represented in NOD/Jax and NOD/ MrkTac mice (figure $1 \mathrm{~F}-\mathrm{J}$ ).

\section{The more diverse microbiota of NOD/MrkTac mice does not rescue NOD/Jax mice from early diabetes}

To test the hypothesis that NOD/MrkTac mice develop diabetes less often and later than NOD/Jax mice because their microbiota is more diverse and more favourably balanced, we transferred via oral gavage $1 \times 10^{10}$ colony-forming units (cfu) of microbes, freshly prepared from caecum contents of NOD/MrkTac mice aged 7-8 weeks, to newly weaned 3 -week-old NOD/Jax mice. In parallel experiments, we cohoused pregnant dams of NOD/Jax colony with dams of NOD/MrkTac colony for 2 weeks before birth of the offspring. This was done to control for potential differences in the adaptation of microbiota at birth and at weaning and to compare the effects of experimental inoculation to vertical transfer occurring under physiological conditions. NOD/Jax mice adopted the microbiota of NOD/MrkTac mice in both experimental approaches (figure $2 \mathrm{~A}-\mathrm{H}$ ) and were colonised by Bacteroidetes including Bacteroides vulgatus,Bacteroides thetaiotaomicron and Bacteroidesfragilis (figure $2 \mathrm{H}$ ) as well as members of genus Parabacteroides (figure 2F) and Prevotella (figure 2G). Principal component analysis (figure 2I) confirmed that the microbiota of
NOD/MrkTac mice had strongly modified the microbiota of NOD/Jax recipients.

In spite of adaptation to donor microbiota composition, neither oral transfer of NOD/MrkTac microbiota at weaning nor its natural transmission by cohousing of pregnant dams rescued $\mathrm{NOD} / J a x$ offspring from developing diabetes at a high incidence (figure 2J,K).

\section{NOD/Jax mice lack Akkermansia and are not colonised with Akkermansia by cohousing}

Both oral gavage and cohousing of NOD/Jax dams with NOD/ MrkTac mice effectively introduced NOD/MrkTac microbiota in $\mathrm{NOD} / \mathrm{Jax}$ mice and improved their microbiota diversity considerably (figure 2). To avoid the premature conclusion that the microbiota would play no role in the development of diabetes in NOD/Jax mice, we scrutinised the $16 \mathrm{~S}$ rRNA sequence data for taxa of low abundance but with potential importance in gut homeostasis. We found a few taxa that were present at low abundancy in NOD/MrkTac microbiota but absent from NOD/ Jax microbiota. These included Mucispirillum, genus AF12 of Rikenellaceae, Odoribacter, C. arthromitus (SFB) and Akkermansia (figure 3). Although to a variable degree, all these taxa were detected by $16 \mathrm{~S}$ rRNA sequencing in NOD/Jax offspring exposed to NOD/MrkTac microbiota (via cohousing or oral gavage) except for Akkermansia (figure 3 and online supplementary figure S2). This suggested that individual members of microbiota not transferred to NOD/Jax mice, including Akkermansia, may defer diabetes development in NOD/MrkTac mice.

\section{A. Muciniphila treatment Delays Diabetes in NOD/Jax Mice, Promotes Segregation Between Bacteria and Epithelium, Lowers Serum Endotoxin Levels and Suppresses Islet TLR Expression and Autoimmunity.}

We hypothesised that if the presence of Akkermansia in NOD/ MrkTac mice plays a role in their delayed diabetes incidence as compared with $\mathrm{NOD} / \mathrm{Jax}$ mice, then oral transfer of Akkermansia to NOD/Jax offspring could similarly delay diabetes development in this model. A. muciniphila is the only mammalian representative of the genus Akkermansia and oral gavage of this symbiont in mice has been found to protect from dietinduced obesity. ${ }^{25} 26$ Using a similar approach, we found that repeated oral gavage of female $\mathrm{NOD} / \mathrm{Jax}$ pups from weaning until 10 weeks of age with $2 \times 10^{8} \mathrm{cfu}$ of $A$. muciniphila delayed diabetes significantly as compared with gavage with vehicle only (figure 4A). By 15 weeks age, only 1 (4\%) of A muciniphilagavaged mice developed diabetes as compared with 11 (44\%) of vehicle-gavaged mice $(\mathrm{P}<0.01)$. Eighty-three per cent of A. muciniphila-gavaged mice were still diabetes free at 18 weeks age, as compared with $52 \%$ of vehicle-gavaged mice $(\mathrm{P}<0.05)$. Gavages with $A$. muciniphila resulted in prompt passage of A. muciniphila to faeces (figure 4B).

On staining the intestinal epithelium for mucus, we found that A. muciniphila administration promoted mucus production by goblet cells in the colon of NOD/Jax mice and reduced crypt depth (figure 4C-F). While reducing the expression of Emr1, a marker of inflammatory and other macrophages, $A$. muciniphila increased that of the anti-inflammatory (type 2) macrophages $Y m 1$, suggesting deviation towards tissue repair functions. In addition, $A$. muciniphila administration tended to increase expression of the antibacterial peptide $\operatorname{Reg} 3 \gamma$, which promotes segregation between microbiota and gut epithelium ${ }^{27}$ (figure 4G). Consistent with this, there was a trend towards increased distance between luminal bacteria and epithelial cells 

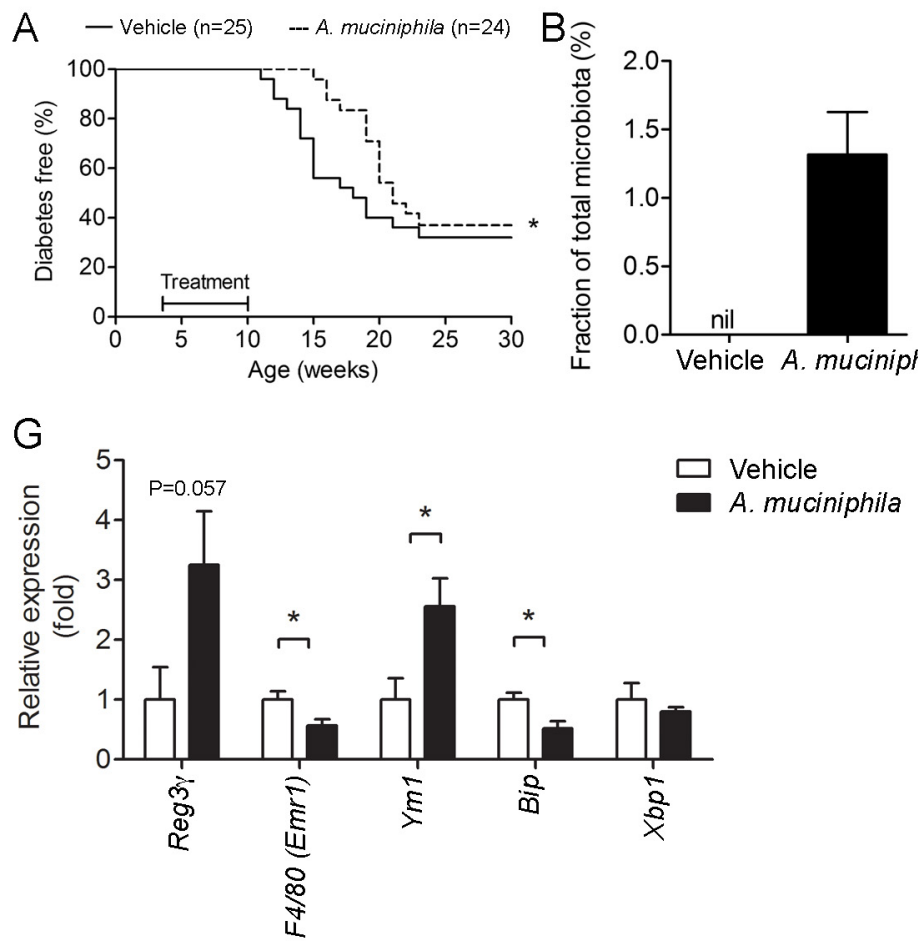
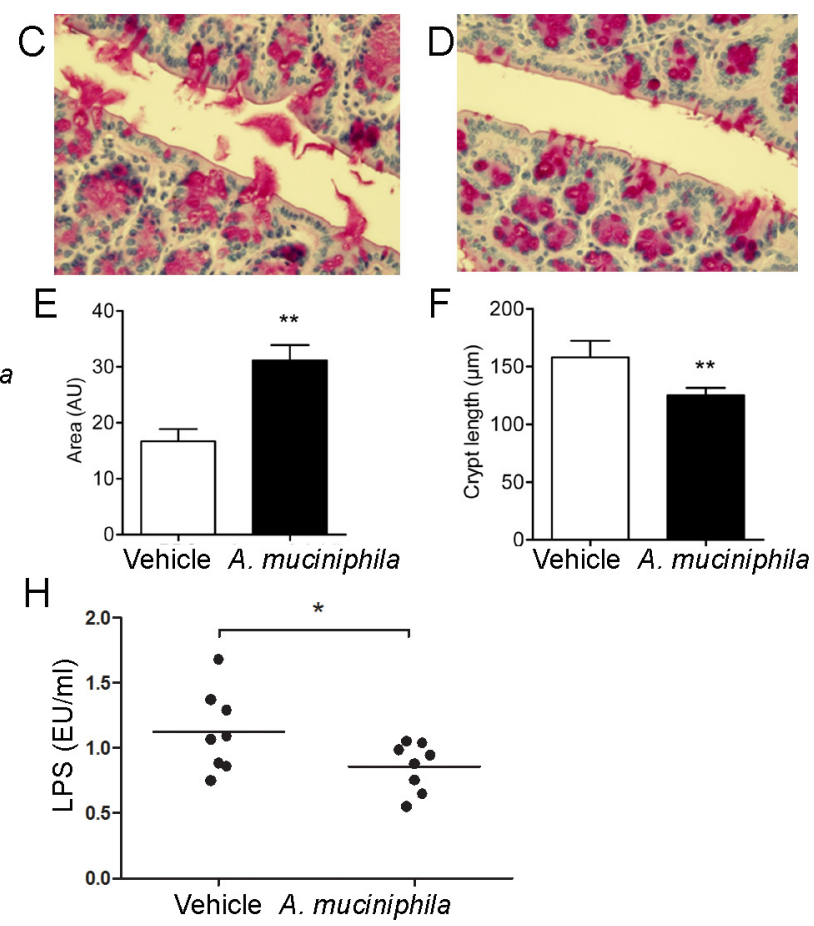

Figure 4 Oral treatment with Akkermansiamuciniphila delays onset of diabetes, promotes epithelial homeostasis, alters gut microbiota composition in NOD/Jax mice and lowers serum endotoxin levels. (A) NOD/Jax mice receiving $A$. muciniphila orally repeatedly at 3 to 10 weeks of age show delayed diabetes incidence as compared with vehicle control $\left({ }^{*} \mathrm{P}<0.05\right.$ for diabetes-free survival and for difference in the proportion of diabetic mice at 18 weeks and $\mathrm{P}<0.01$ for difference at 15 weeks). (B) Comparison of $A$. muciniphila levels in the microbiota of $A$. muciniphila-treated and vehicle-treated mice based on 16S rRNA sequencing of total microbiota. (C-E) PAS staining of colon sections from A. muciniphila-treated (C) and vehicle-treated (D) mouse showing mucus (red) in gut lumen. (E) Relative area of mucus in the lumen of transverse colon determined from mice ( $\mathrm{n}=6$ per group) analysed from micrographs such as in (C) and D). (F) Effect of $A$. muciniphila treatment on crypt length expressed as the mean thickness in 10-20 visual fields per mouse ( $\mathrm{n}=5$ mice/group). (G) Expression of transcripts of antibacterial peptide Reg3 $\gamma$, macrophage markers F4/80 and Ym1 (total and M2) and stress-response markers Bip and Xbp1 in the colon ( $n=6$ per group). (H) Levels of the bacterial endotoxin LPS in sera of mice treated with A. muciniphila or vehicle. The results shown in (B)-(H) are from 7-week-old mice treated for 4 weeks. LPS, lipopolysaccharide; NOD, nonobese diabetic mice. PAS, periodic acid-Schiff.

(online supplementary figure S3). Expression of the immunoglobulin binding protein BiP was downregulated and a trend was seen for downregulation of X-box binding protein- $1 \mathrm{Xbp} 1,{ }^{28}$ both sensors of - endoplasmic reticulum-associated stress in intestinal epithelial cells (figure 4G). These epithelial homeostasis-related phenomena in the gut also reflected beneficially to endotoxin levels in the serum, which were significantly lower in A. muciniphila-treated mice (figure $4 \mathrm{H}$ ).

In pancreatic islets, A. muciniphila treatment diminished TLR2 and TLR4 levels, increased the number of regulatory Foxp $3^{+}$Treg cells and reduced overall infiltration of mononuclear leukocytes in islets (figure 5A-E). Transcripts of Treg-associated cytokines IL-10 and TGF- $\beta$ were also elevated in pancreas-draining lymph node (figure $5 \mathrm{~F}, \mathrm{G}$ ), which is proposed to respond to innate immune signals from the colon. ${ }^{29} 30 \mathrm{~A}$ reduced dose of $A$. muciniphila also diminished serum endotoxin levels and islet infiltration by mononuclear cells (online supplementary figure S4).

\section{A.muciniphila treatment promotes remodelling of microbiota in NOD/Jax mice}

Although the observed effects on gut homeostasis can be ascribed to the effects of $A$. muciniphila colonisation itself, it was important to consider potential effects of the treatment on mutualism with other species and taxa. A trend towards reduction in Firmicutes and Clostridia (figure 6A) was consistent with the difference between NOD/Jax and NOD/MrkTac microbiota. On species level, a significant reduction was observed of Ruminococcus torques (figure 6B,C).

\section{DISCUSSION}

There is a growing body of evidence supporting an association between the intestinal microbiota and T1D, in spite of differences in technological platforms and clinical data sets. ${ }^{37}$ However, the strength of this association and the identified microbial taxa are highly variable, and more importantly, no causality has yet been shown. In this study, we use the NOD mouse model of T1D and embark on the observation that microbiota composition was strikingly different in two parallel colonies of NOD mice from different suppliers and with different diabetes incidence. ${ }^{31}$ Using deep microbiota analysis, cohousing and inoculation transfer experiments, as well as single strain administration, we rule out the potential impact of some canonical intestinal microbes and provide evidence for the causal effect of the mucosal symbiont A. muciniphila in delaying the onset and development of T1D.

The characterisation by deep $16 \mathrm{~S}$ rRNA sequencing of the intestinal microbiota from NOD/MrkTac and NOD/Jax mice, with high and low diabetes incidence, respectively, revealed surprising differences. The alpha diversity of NOD/Jax microbiota was significantly reduced as compared with NOD/MrkTac 
A
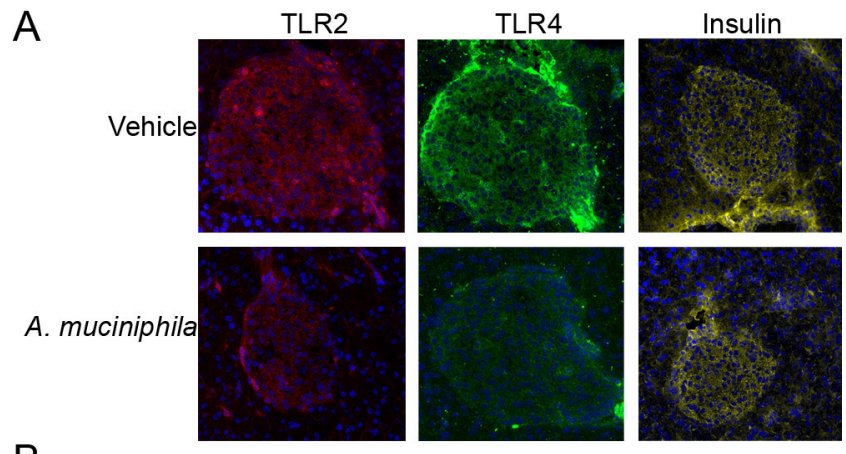

$\mathrm{B}$
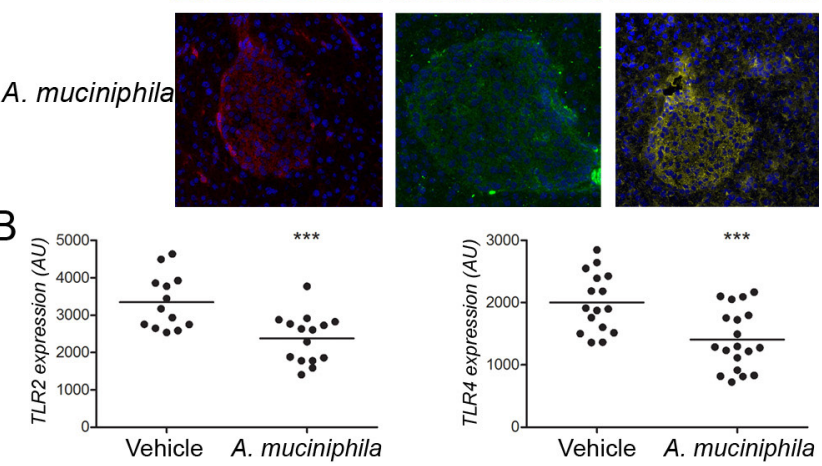

C
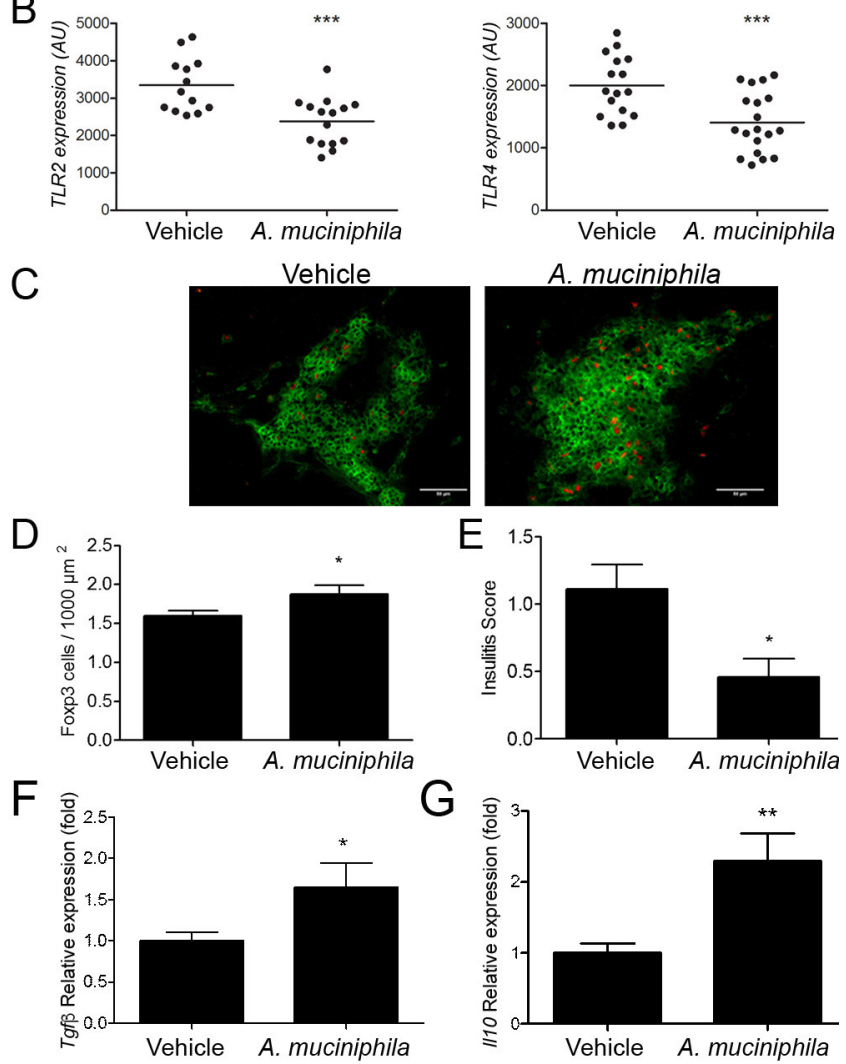

$\mathrm{G}$

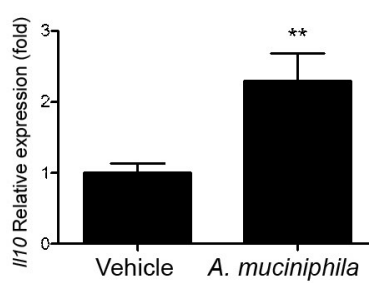

Figure 5 A 4-week Akkermansia muciniphila treatment with oral $A$. muciniphila reduces TLR2 and TLR4 expression, increases the number of Foxp $3^{+}$Treg cells in pancreatic islets and promotes regulatory cytokines TGF- $\beta$ and IL-10 in pancreatic lymph node. Pancreas cryosections from A. muciniphila-treated and (B) vehicle-treated mice were stained for insulin, TLR2 and TLR4 (A, B) and for Foxp3 (red) and CD4 (green) (C) A. muciniphila. (D) The number of Foxp $3^{+}$Treg (red dots) was calculated relative to the area of CD4 T-cell infiltrate (green) in each islet. (E) Insulitis scores in A. muciniphila-treated and vehicle-treated mice. Relative expression of (F) Tgf $\beta$ and (G) IL-10 transcripts in pancreatic lymph node normalised to $\beta$-actin. Data are as mean $\pm S E M n=4$ mice/ group analysed at 7 weeks of age. ${ }^{*} \mathrm{P}<0.05$; ${ }^{* *} \mathrm{P}<0.01$; ${ }^{* * *} \mathrm{P}<0.001$. IL-10, interleukin 10; TGF- $\beta$, transforming growth factor beta.

microbiota, and although Bacteroidetes were relatively sparse in both colonies, the Bacteroidetes-to-Firmicutes ratio was significantly lower in NOD/Jax mice due to almost complete lack of Bacteroides, Prevotella and Parabacteroides in these mice. A reduced diversity of microbiota is thought to be associated with reduced resistance against microbial and inflammatory imbalance and predispose to loss of immune tolerance. In children with increased genetic susceptibility to T1D, loss of diversity due to increase in bacteria related to Blautia, Rikenellaceae
A
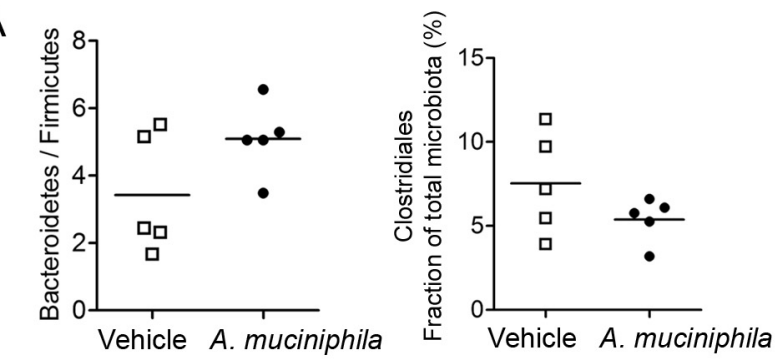

$\mathrm{B}$

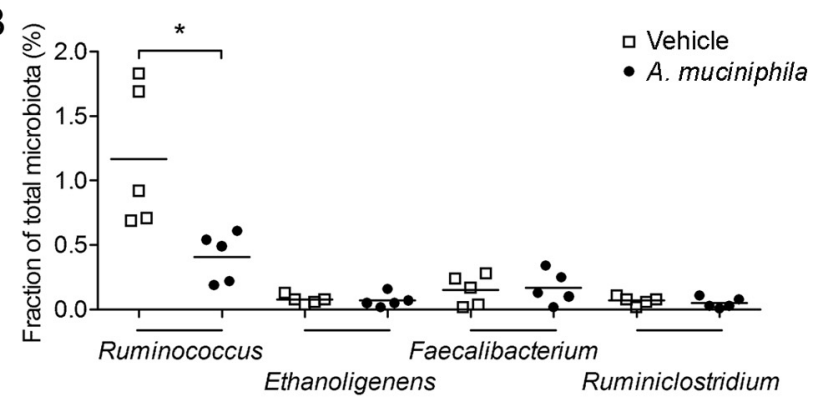

C

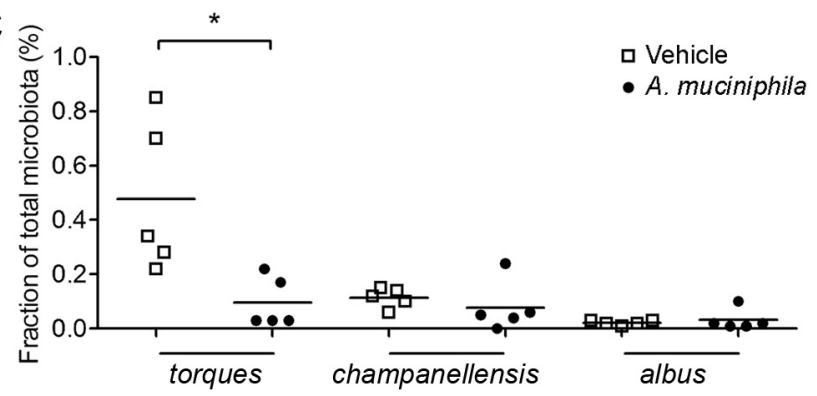

Figure 6 A 4-week treatment with oral Akkermansia muciniphila induces microbiota remodelling and outcompetes Ruminococcus torques. (A) Bacteroidetes-to-Firmicutes ratio and relative abundance of Clostridiales (B) and of representative genera of Clostridiaceae and (C) of three species of Ruminococcus in A. muciniphila-treated and PBS-treated mice. Data are as mean \pm SEM. PBS, phosphate buffered saline.

and Ruminococcus was recently found to correlate with risk to progress to clinical diabetes. ${ }^{3}$ Moreover, the abundance of Bacteroides species has been associated with the appearance of diabetes-related autoantibodies. ${ }^{2} 2232$ Loss of immune tolerance due to substantial overgrowth of Bacteroidetes was recently proposed to associate with the inferior capacity of LPS/endotoxin of Bacteroides dorei to establish LPS tolerance. ${ }^{7}$ On the other hand, $B$. fragilis and other Bacteroides species induce IL-10-secreting and Foxp3 expressing regulatory $\operatorname{Tr} 1$ and Treg cells in gnotobiotic mice and suppress induction of inflammatory colitis. ${ }^{1718}$ Association of Bacteroides with diabetes development in different animal models of T1D is less clear. In one study, Bacteroides spp were found to be more abundant in young NOD mice which did not develop diabetes. ${ }^{33}$ In this study, Bacteroides spp abundance associated with low-diabetes incidence and revealed the presence of species potentially supporting the establishment of immune tolerance in the NOD/MrkTac colony, such as $B$. fragilis and $B$. thetaiotaomicron ${ }^{13} 1417^{18}$ (see also online supplementary discussion).

Metagenomic sequencing of gut microbiota using recently developed bioinformatics approaches ${ }^{34}$ has revealed an increase in inflammation-associated species and metabolic pathways in children who developed T1D. ${ }^{3}$ Conversely, microbiota in 
children who did not develop autoantibodies was characterised by an abundancy of butyrate-producing and mucin-degrading bacteria, including Prevotella and Akkermansia. ${ }^{21}$ In NOD mice, increased levels of A. muciniphila associated with reduced diabetes incidence following vancomycin treatment after birth. ${ }^{35}$ In line with this, we detected A. muciniphila by $16 \mathrm{~S}$ rRNA sequencing only in mice in the NOD/MrkTac colony, and in our colonisation experiments, there was no transfer of A. muciniphila to NOD/Jax mice. The observed failure of A. muciniphila to colonise recipient mice during cohousing could have various causes, including its known sensitivity to atmospheric oxygen ${ }^{19}$ amplified by its presence on the outer surface of the mouse faecal pellets or a limited competitiveness of A. muciniphila, requiring repeated transfers.

The type strain A. muciniphila $\mathrm{Muc}^{\mathrm{T}}$ is the sole publicly available cultured Akkermansia species from the human intestinal tract, encoding a wide array of mucin-degrading enzymes and producing propionate and acetate, the latter of which can be converted to butyrate by several human butyrogens. ${ }^{36}{ }^{37}$ The levels of A. muciniphila correlate inversely with the incidence of IBD, ${ }^{38}$ metabolic syndrome ${ }^{39} 40$ and acute appendicitis, ${ }^{41}$ suggesting that $A$. muciniphila has metabolic and immune-modifying effects which promote gut homeostasis and regulate inflammatory disorders. ${ }^{42}$ Recently, an association between A. muciniphila levels and clinical response to immune checkpoint inhibitor PD-1 was identified. ${ }^{43}$ Because repeated gavage of A. muciniphila strain $\mathrm{Muc}^{\mathrm{T}}$ resulted in an impact in dietinduced obesity in mice, ${ }^{25} 26$ we tested if repeated gavages with A. muciniphila would exert diabetes-retarding effects in NOD mice. Although selection of a common gut bacteria such as Escherichia coli or Lactobacillus spp to gavage the control group might have addressed some aspects of the specificity of A. muciniphila treatment, the inherent uncertainty of species or strain variation in their potential effects, as exemplified by the reported effects of a strain of E. coli (Nissle strain) ${ }^{44}$ or Lactobacillus casei or Lactobacillus johnsoni $i^{45}{ }^{46}$ prompted us to use the vehicle alone as the control treatment similarly to earlier work. ${ }^{2526}$ While nearly half of vehicle-gavaged mice were diabetic by 15 weeks age, only one of A. muciniphila-gavaged mice had developed diabetes. Diabetes-free survival was significantly enhanced by $A$. muciniphila gavages until 20 weeks age, after which the effect of A. muciniphila gradually waned when compared with controls. This was apparent especially because diabetes-free survival in vehicle-gavaged mice remained higher than in control mice in other experiments, likely reflecting the increased interfering in these experiments that the mice were subjected to. Extending the gavages beyond 10 weeks of age to test if the difference in diabetes-free survival would sustain was therefore not further considered.

Administration of A. muciniphila to NOD/Jax mice tended to increase expression of the antibacterial peptide Reg3 $\gamma$, involved in maintaining spatial segregation of microbiota from host in the intestine ${ }^{27}$ and previously shown to be upregulated by transfer of A. muciniphila. ${ }^{25}$ The present observations are in line with recent data obtained with $\mathrm{C} 57 \mathrm{BL} / 6 \mathrm{~J}$ mice that also showed a $32-\mathrm{kD}$ protein located in the outer membrane of A. muciniphila to increase barrier function, likely via its signalling to the TLR2 receptor. ${ }^{26}$ In view of the evidence that inflammatory stress in the colon promotes autoimmune activity in pancreatic lymph node, lymphocyte accumulation in islets and development of diabetes in NOD mice, ${ }^{3047-49}$ we envision that one mechanism by which A. muciniphila reduces diabetogenesis in NOD mice is by increasing the thickness of the mucus layer and antagonising its colonisation by inflammatory gut pathobionts, therewith lowering systemic endotoxin levels and TLR2 and TLR4 levels in islets. ${ }^{50}$ One such pathobiont is $R$. torques. It is 100 -fold more abundant in the mucosa in patients with Crohn's disease and ulcerative colitis than in healthy subjects. ${ }^{38} \mathrm{~A}$ trade-off between these two mucolytic species was observed in a dietary intervention trial in Crohn's disease patients ${ }^{51}$ similar to that observed in our NOD mice following A. muciniphila transfer, supporting this mechanistic explanation. It remains to be studied whether this competition is mediated by $\operatorname{Reg} 3 \gamma$ that is induced by A. muciniphila administration and is known to inhibit Gram-positive bacteria, such as $R$. torques or that both mucolytic species share the same niche and that orally transferred A. muciniphila is able to compete out $R$. torques. Recently, A. muciniphila was found to associate with diminished risk of developing T1D-associated autoantibodies in children in two separate studies, ${ }^{21}{ }^{23}$ suggesting that this symbiont may promote immunoregulation during early pathogenesis of T1D also in humans.

In summary, a high-incidence NOD colony was not protected by transfer of microbiota from a low-incidence mouse colony, although this led to major changes in intestinal taxa. This suggests that the development of autoimmune diabetes is not necessarily regulated by the overall balance of microbial taxa in gut microbiota but by individual microbes which interact with the host as well as other members of microbiota. One of these taxa is the intestinal symbiont $A$. muciniphila, which evokes metabolic and immune signalling to promote immune tolerance in the gut and beyond. This may reflect beneficially to the control of autoimmune diseases such as T1D and open new possibilities for their treatment.

\section{MATERIALS AND METHODS \\ Mice}

NOD/MrkTac and NOD/Jax mice were obtained from Taconic and Jackson laboratories, respectively. Mice were bred and maintained in Turku University's central animal facility under specific pathogen-free housing conditions and under filter lids. To avoid transfer of microbiota between colonies, NOD/Jax mice were kept in a separate unit not visited during the same day of visiting the unit in which NOD/MrkTac mice were kept. All experiments were approved by the national board of animal experimentation in Finland under license ESAVI/6082/04.10.07/2014.

\section{Microbiota analysis}

Microbiota was profiled by Illumina MiSeq sequencing of $16 \mathrm{~S}$ rRNA amplicons obtained from bacterial DNA, isolated from stools or caecal and colon contents, as reported previously using primers for V4-V5 or V3-V4 variable regions. ${ }^{49}$ Data were analysed using QIIME software with Greengenes database (2013 updated version) and to increase species-level identifications, using Kraken alignment tool. ${ }^{52}$

For initial profiling of the microbiota in NOD/MrkTac and NOD/Jax mice, bacterial DNA from caecal and colon contents was also amplified by genus or group-specific primers, including Bacteroides spp, Clostridia groups IV and XIVa, Bifidobacteriaceae, lactobacilli and Coriobacteriaceae, as reported previously and performed by Alimetrics (Espoo, Finland). ${ }^{49}$

\section{Cohousing and oral transfer of microbiota}

In separate experiments, NOD/Jax mice were exposed to NOD/ MrkTac microbiota either by cohousing or by oral transfer of bacteria. For cohousing, pregnant NOD/Jax dams were put together with NOD/MrkTac female mice for 14 days, and the mice were again separated before NOD/Jax dams gave birth. For 
oral microbiota transfer, caecal and colon contents of two NOD/ MrkTac female donors (or NOD/Jax donors) were pooled and dissolved in anaerobic PBS and the total bacteria were collected from the supernatant after gentle centrifugation at low speed to pellet all solid material. The bacterial suspension was adjusted to $2 \mathrm{~mL}$ volume with PBS. Female NOD/Jax pups from several litters were then allocated to two groups which were orally gavaged with $330 \mu \mathrm{L}$ of bacterial suspension containing $1 \times 10^{10}$ bacteria of either NOD/MrkTac or NOD/Jax female origin twice with 3-day interval.

\section{Oral feeding protocol of cultured $A$. muciniphila}

A. muciniphila strain $\mathrm{Muc}^{\mathrm{T}}$ (CIP $107961^{\mathrm{T}}$ ) was cultured under strictly anaerobic conditions as described previously, ${ }^{24}$ and exponentially growing cultures were washed with PBS and immediately frozen in PBS containing 25\% glycerol to a final concentration of $10^{10}$ cells per $\mathrm{mL} .^{25}$ Prior to administration, a frozen pellet of A. muciniphila was thawed, resuspended in anaerobic PBS to a concentration of $1 \times 10^{9}$ per mL. NODJax pups were fed three times a week with $2 \times 10^{8} \mathrm{cfu}$ starting from 3 weeks of age until the mice reached 10 weeks of age. In one experiment, a group of mice was treated with 1:100 part of the original dose (ie, $2 \times 10^{6} \mathrm{cfu}$ ) in parallel with groups treated with the original dose and vehicle as control.

\section{Determination of diabetes and serum endotoxin levels}

In follow-up experiments, diabetes-free survival was determined by weekly measurements of blood glucose concentrations. Minute samples were drawn from the tail vein and blood glucose was immediately assessed with quick blood glucose meter (Contour, Bayer, Germany). A value equal or above $12.0 \mathrm{mmol} / \mathrm{L}$ in two consecutive readings was used for definition of diabetes. Serum samples were prepared from blood aliquots withdrawn via cardiac puncture. Endotoxin levels were measured using Pierce LAL Chromogenic Endotoxin Quantitation Kit (Thermo Scientific).

\section{Gut histology and in situ hybridisation}

Gut homeostasis and the effects of $A$. muciniphila transfers on gut were evaluated from tissue samples prepared from NOD/ MrkTac and NOD/Jax mice/A. muciniphila-treated NOD/Jax mice at 7 weeks of age using Carnoy's solution as a fixative. Briefly, parts of transverse colon were processed for histology and stained with H\&E or with periodic acid-Schiff for determination of crypt length and mucus production.

For evaluating the proximity of luminal bacteria to villous surfaces, sections of transverse colon were processed for in situ hybridisation according to a previously published method. ${ }^{53}$ Briefly, luminal bacteria were labelled with Alexa Fluor 647-conjugated eubacterial probe EUB-338 (5๑-GCTGCCTCCCGTAGGAGT-3 $\square$, Invitrogen, Carlsbad, CA) in Tris- $\mathrm{HCl}$ buffer $(20 \mathrm{mM}$ Tris- $\mathrm{HCl}, \mathrm{pH} 7.4,0.9 \mathrm{M} \mathrm{NaCl}, 0,1 \%$ sodium dodecyl sulfate, $20 \%$ formamide). Mucus was stained with an antibody to mucin 2 (rabbit antimuc2, Santa Cruz Biotechnology, Santa Cruz, CA, USA) and antirabbit Alexa Fluor 488 (Abcam, USA).

\section{Determination of cytokine and stress-related transcripts with quantitative PCR}

Transverse colon and pancreatic lymph nodes were dissected from 7-week-old mice and stored in RNA later (Qiagen, Germantown, MD, USA). Samples were processed and analysed by quantitative PCR as described earlier ${ }^{49}$ (for specific primers and transcripts analysed, see online supplementary table 1).

\section{Pancreas histology and immunohistochemistry}

Pancreases from 7-week-old mice treated with oral A. muciniphila were snap frozen in liquid nitrogen, frozen at $-70 \mathrm{C}^{\circ}$ and cryosections fixed in ice-cold acetone were stained with the following antibodies: polyclonal rabbit anti-TLR2 (ThermoFisher), anti-TLR4 (Abcam), anti-insulin (Santa Cruz Biochemicals) and as the second-step antibody to each of these, Alexa488-conjugated or Alexa647-conjugated goat antirabbit $\operatorname{IgG}(\mathrm{H}+\mathrm{L})$ (Invitrogen) or Alexa488-conjugated antimouse CD4 (Biolegend) and eFluor615-conjugated antimouse/ rat Foxp3 (eBioscience). Photographs for TLR2, TLR4 and insulin expression were taken with Nikon Eclipse Ti-2 microscope and Hamamatsu digital camera. Photographs for CD4 and Foxp3 expression were taken with Nikon Ci-L Plan Fluor CoolLED microscope using DS-Fi2 camera. TLR2 and TLR4 expression levels were quantified using ImageJ software (a free software supported by NIH, USA). Insulitis scores were evaluated from H\&E-stained sections of each pancreas. Each islet was scored (0-3) depending on the area occupied by leucocyte infiltrates (50-70 islets/pancreas).

\section{Statistical analyses}

Differences in diabetes incidence were evaluated by survival analysis (Gehan-Breslow-Wilcoxon test) and by comparison of the proportion of diabetic mice at 15 and 18 weeks of age (Fisher's exact test). Results with numerical values were analysed for statistical significance using unpaired Student's t-test and analysis of variance.

Acknowledgements We are grateful to Steven Aalvink and Satu Silojärvi for expert technical assistance.

Contributors $\mathrm{AH}$ and WMDV designed the work; $\mathrm{AH}$ analysed data and wrote the first version of the manuscript; RT, SAP, CB, HP, PDC, JPO and RE performed experiments and/or analysed data; WMDV and PDC reviewed the manuscript and wrote parts of the final version, which all authors approved.

Funding This work was supported by Päivikki and Sakari Sohlberg Foundation (AH), Foundation for Diabetes Research Finland (AH), FRFS-WELBIO (WELBIO-CR-2012S02R) (PDC), Funds Baillet Latour (PDC), European Commission (250172) (PDC), Horizon 2020 Framework Programme of European Research Council (336452) (PDC), H2020 Excellent Science and H2020 European Research Council (250172) (WMDV), Netherlands Organization for Scientific Research (024.002.002) (WMDV), Academy of Finland (1272870, 137389, 141140 (WMDV) and 285503 (AH)).

Competing interests None declared.

Provenance and peer review Not commissioned; externally peer reviewed.

(c) Article author(s) (or their employer(s) unless otherwise stated in the text of the article) 2018. All rights reserved. No commercial use is permitted unless otherwise expressly granted.

\section{REFERENCES}

1 Yurkovetskiy LA, Pickard JM, Chervonsky AV. Microbiota and autoimmunity: exploring new avenues. Cell Host Microbe 2015;17:548-52.

2 Davis-Richardson AG, Ardissone AN, Dias R, et al. Bacteroides dorei dominates gut microbiome prior to autoimmunity in Finnish children at high risk for type 1 diabetes. Front Microbiol 2014;5:678.

3 Kostic AD, Gevers D, Siljander $H$, et al. The dynamics of the human infant gut microbiome in development and in progression toward type 1 diabetes. Cell Host Microbe 2015:17:260-73.

4 Davis-Richardson AG, Triplett EW. A model for the role of gut bacteria in the development of autoimmunity for type 1 diabetes. Diabetologia 2015;58:1386-93.

5 Hänninen AL, Toivonen RK. On the role of gut bacteria and infant diet in the development of autoimmunity for type 1 diabetes. Diabetologia 2015;58:2195-6.

6 Chervonsky AV. Microbiota and autoimmunity. Cold Spring Harb Perspect Biol 2013;5:a007294.

7 Vatanen T, Kostic AD, d'Hennezel E, et al. Variation in microbiome LPS immunogenicity contributes to autoimmunity in humans. Cell 2016;165:842-53. 
8 Gülden E, Ihira M, Ohashi A, et al. Toll-like receptor 4 deficiency accelerates the development of insulin-deficient diabetes in non-obese diabetic mice. PLoS One 2013;8:e75385.

9 Brown K, Godovannyi A, Ma C, et al. Prolonged antibiotic treatment induces a diabetogenic intestinal microbiome that accelerates diabetes in NOD mice. Isme J 2016;10:321-32.

10 Chow J, Mazmanian SK. Getting the bugs out of the immune system: do bacterial microbiota "fix" intestinal T cell responses? Cell Host Microbe 2009:5:8-12.

11 Cox LM, Yamanishi S, Sohn J, et al. Altering the intestinal microbiota during a critical developmental window has lasting metabolic consequences. Cell 2014;158:705-21.

12 Hooper LV, Littman DR, Macpherson AJ. Interactions between the microbiota and the immune system. Science 2012;336:1268-73.

13 Atarashi K, Tanoue T, Oshima K, et al. Treg induction by a rationally selected mixture of Clostridia strains from the human microbiota. Nature 2013:500:232-6.

14 Atarashi K, Tanoue T, Shima T, et al. Induction of colonic regulatory T cells by indigenous Clostridium species. Science 2011;331:337-41.

15 Atarashi K, Tanoue T, Ando M, et al. Th17 cell induction by adhesion of microbes to intestinal epithelial cells. Cell 2015;163:367-80.

16 Ivanov II, Frutos RL, Manel N, et al. Specific microbiota direct the differentiation of IL-17-producing T-helper cells in the mucosa of the small intestine. Cell Host Microbe 2008:4:337-49.

17 Mazmanian SK, Round JL, Kasper DL. A microbial symbiosis factor prevents intestinal inflammatory disease. Nature 2008:453:620-5.

18 Round JL, Mazmanian SK. Inducible Foxp3+ regulatory T-cell development by a commensal bacterium of the intestinal microbiota. Proc Natl Acad Sci U SA 2010;107:12204-9.

19 Derrien M, Vaughan EE, Plugge CM, et al. Akkermansia muciniphila gen. nov., sp. nov., a human intestinal mucin-degrading bacterium. Int J Syst Evol Microbiol 2004;54(Pt 5):1469-76.

20 Shin NR, Lee JC, Lee HY, et al. An increase in the Akkermansia spp. population induced by metformin treatment improves glucose homeostasis in diet-induced obese mice. Gut 2014:63:727-35.

21 Brown CT, Davis-Richardson AG, Giongo A, et al. Gut microbiome metagenomics analysis suggests a functional model for the development of autoimmunity for type 1 diabetes. PLoS One 2011;6:e25792.

22 de Goffau MC, Fuentes S, van den Bogert B, et al. Aberrant gut microbiota composition at the onset of type 1 diabetes in young children. Diabetologia 2014;57:1569-77.

23 Endesfelder D, Engel M, Davis-Richardson AG, et al. Towards a functional hypothesis relating anti-islet cell autoimmunity to the dietary impact on microbial communities and butyrate production. Microbiome 2016;4:17

24 Derrien M, Van Baarlen P, Hooiveld G, et al. Modulation of mucosal immune response, tolerance, and proliferation in mice colonized by the mucin-degrader akkermansia muciniphila. Front Microbiol 2011;2:166.

25 Everard A, Belzer C, Geurts L, et al. Cross-talk between Akkermansia muciniphila and intestinal epithelium controls diet-induced obesity. Proc Natl Acad Sci U SA 2013;110:9066-71.

26 Plovier H, Everard A, Druart C, et al. A purified membrane protein from Akkermansia muciniphila or the pasteurized bacterium improves metabolism in obese and diabetic mice. Nat Med 2017;23:107-13.

27 Vaishnava S, Yamamoto M, Severson KM, et al. The antibacterial lectin Reglllgamma promotes the spatial segregation of microbiota and host in the intestine. Science 2011;334:255-8.

28 Niederreiter L, Fritz TM, Adolph TE, et al. ER stress transcription factor Xbp1 suppresses intestinal tumorigenesis and directs intestinal stem cells. J Exp Med 2013:210:2041-56.

29 Toivonen R, Kong L, Rasool O, et al. Activation of plasmacytoid dendritic cells in colon-draining lymph nodes during citrobacter rodentium infection involves pathogensensing and inflammatory pathways distinct from conventional dendritic cells. J Immunol 2016:196:4750-9.
30 Turley SJ, Lee JW, Dutton-Swain N, et al. Endocrine self and gut non-self intersect in the pancreatic lymph nodes. Proc Natl Acad Sci U S A 2005;102:17729-33.

31 Pozzilli P, Signore A, Williams AJ, et al. NOD mouse colonies around the world-recent facts and figures. Immunol Today 1993:14:193-6.

32 Murri M, Leiva I, Gomez-Zumaquero JM, et al. Gut microbiota in children with type 1 diabetes differs from that in healthy children: a case-control study. BMC Med 2013;11:46

33 Krych $\nvdash$, Nielsen DS, Hansen AK, et al. Gut microbial markers are associated with diabetes onset, regulatory imbalance, and IFN- $\gamma$ level in NOD mice. Gut Microbes 2015;6:101-9.

34 Luo C, Knight R, Siljander $H$, et al. ConStrains identifies microbial strains in metagenomic datasets. Nat Biotechnol 2015;33:1045-52.

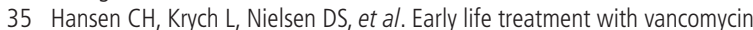
propagates Akkermansia muciniphila and reduces diabetes incidence in the NOD mouse. Diabetologia 2012;55:2285-94.

36 Belzer C, de Vos WM. Microbes inside-from diversity to function: the case of Akkermansia. Isme J 2012:6:1449-58.

37 van Passel MW, Kant R, Zoetendal EG, et al. The genome of Akkermansia muciniphila, a dedicated intestinal mucin degrader, and its use in exploring intestinal metagenomes. PLoS One 2011;6:e16876.

38 Png CW, Lindén SK, Gilshenan KS, et al. Mucolytic bacteria with increased prevalence in IBD mucosa augment in vitro utilization of mucin by other bacteria. Am J Gastroenterol 2010;105:2420-8.

39 Santacruz A, Collado MC, García-Valdés L, et al. Gut microbiota composition is associated with body weight, weight gain and biochemical parameters in pregnant women. Br J Nutr 2010;104:83-92.

40 Zhang $\mathrm{H}$, DiBaise JK, Zuccolo A, et al. Human gut microbiota in obesity and after gastric bypass. Proc Natl Acad Sci U S A 2009;106:2365-70.

41 Swidsinski A, Dörffel Y, Loening-Baucke V, et al. Acute appendicitis is characterised by local invasion with Fusobacterium nucleatum/necrophorum. Gut 2011;60:34-40.

42 Derrien M, van Passel MW, van de Bovenkamp JH, et al. Mucin-bacterial interactions in the human oral cavity and digestive tract. Gut Microbes 2010;1:254-68.

43 Routy B, Le Chatelier E, Derosa L, et al. Gut microbiome influences efficacy of PD-1based immunotherapy against epithelial tumors. Science 2017:eaan3706.

44 Schultz M. Clinical use of E. coli Nissle 1917 in inflammatory bowel disease. Inflamm Bowel Dis 2008;14:1012-8

45 Lau K, Benitez P, Ardissone A, et al. Inhibition of type 1 diabetes correlated to a Lactobacillus johnsonii N6.2-mediated Th17 bias. J Immunol 2011;186:3538-46.

46 Matsuzaki T, Nagata Y, Kado S, et al. Prevention of onset in an insulin-dependent diabetes mellitus model, NOD mice, by oral feeding of Lactobacillus casei. APMIS 1997; 105:643-9.

47 Alam C, Valkonen S, Palagani V, et al. Inflammatory tendencies and overproduction of $\mathrm{IL}-17$ in the colon of young NOD mice are counteracted with diet change. Diabetes 2010:59:2237-46.

48 Lee AS, Gibson DL, Zhang Y, et al. Gut barrier disruption by an enteric bacterial pathogen accelerates insulitis in NOD mice. Diabetologia 2010;53:741-8.

49 Toivonen RK, Emani R, Munukka E, et al. Fermentable fibres condition colon microbiota and promote diabetogenesis in NOD mice. Diabetologia 2014;57:2183-92.

50 Wen $\mathrm{L}$, Peng J, Li Z , et al. The effect of innate immunity on autoimmune diabetes and the expression of Toll-like receptors on pancreatic islets. J Immunol 2004;172:3173-80

51 Halmos EP, Christophersen CT, Bird AR, et al. Consistent prebiotic effect on gut microbiota with altered FODMAP intake in patients with Crohn's disease: a randomised, controlled cross-over trial of well-defined diets. Clin Trans/ Gastroenterol 2016;7:e164

52 Kuczynski J, Stombaugh J, Walters WA, et al. Using QIIME to analyze 16S rRNA gene sequences from microbial communities. Curr Protoc Bioinformatics 2011; Chapter 10:Unit 10.7.

53 Chassaing B, Koren O, Goodrich JK, et al. Dietary emulsifiers impact the mouse gut microbiota promoting colitis and metabolic syndrome. Nature 2015:519:92-6. 